\title{
OBSERVACIONES ECOLÓGICAS Y TAXONOMÍA DEL GÉNERO Codonanthe (Mart.) Hanst. (GESNERIACEAE) EN LA CUENCA DEL RÍO LOS AMIGOS, MADRE DE DIOS - PERÚ
}

\section{ECOLOGICAL OBSERVATIONS AND TAXONOMY OF THE GENUS Codonanthe (Mart.) Hanst. (GESNERIACEAE) AT THE LOS AMIGOS RIVER BASIN (MADRE DE DIOS - PERU)}

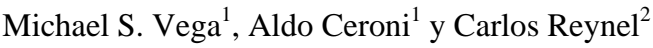

\section{Resumen}

Se realizaron colectas de especimenes del género Codonanthe (Mart.) Hanst. en los tres principales tipos de bosques de la cuenca del río Los Amigos: aguajal, bosque de llanura baja o "bajío" y bosque de terraza alta. Se encontraron dos especies: C. crassifolia y C. uleana; ambas de hábito epífito, y de las cuales $C$. uleana fue encontrada usualmente asociadas a hormigas (“jardines de hormigas”) de las especies Azteca sp., Camponotus femoratus y Crematogaster cf limata parabiotica. De acuerdo al tipo de hábitat y a la cantidad de luz que recibían las plantas se observaron variaciones en la abundancia y morfología de estas especies de epífitas. La especie $C$. crassifolia es un nuevo reporte para la flora de Madre de Dios.

Palabras Claves: Codonanthe, epífitas, jardines de hormigas, interacciones parabióticas, epifitismo

\begin{abstract}
We present observations and collections of specimens of the genus Codonanthe (Mart.) Hanst. from the three main types of forests at Los Amigos River: aguajal, plain low forest or "bajío" and high terrace forest. We found two species: C. uleana and C. crassifolia; both of epiphytic habit; $C$. uleana was usually found associated with ants (“ant gardens”): Azteca sp., Camponotus femoratus and Crematogaster cf limata parabiotica. According to the type of habitat and to the amount of light received, we observed variations in the abundance and morphology of these epiphytic species. C. crassifolia is a new report to the flora of Madre de Dios.
\end{abstract}

Key Words: Codonanthe, epiphyts, ant gardens, parabiotic interactions, epiphytism

\section{Introducción}

La familia Gesneriaceae es componente importante de los bosques tropicales con mucho potencial ornamental, pero con serios problemas de conservación debido a la destrucción de su hábitat (Wiehler, 1983; Kvist et al., 2004; Hernani, 2006). En el Perú esta familia cuenta con aproximadamente 137 especies y 30 géneros (Brako \& Zarucchi, 1993; Ulloa et al., 2004), de las cuales 60 especies son endémicas. Aunque existen muchas especies de hierbas y algunas arbustivas dentro de esta familia las Gesneriáceas están consideradas dentro de las 10 familias con más especies epífitas entre las dicotiledóneas, con aproximadamente un $20 \%$ de epifitismo dentro del total de las especies (Wiehler, 1983; Hernani, 2006).

El género Codonanthe está formado por plantas de hábito principalmente epífito, desarrollando adaptaciones de reserva, de allí que las hojas son suculentas. El género es completamente Neotropical y cuenta con cerca de 15 especies distribuidas desde México hasta Brasil y Bolivia, también en las Antillas Menores. En el Perú se reportan 3 especies: $C$. corniculata Wiehler, reportada en Loreto y San Martín hasta los $1000 \mathrm{msnm}$; C. uleana Fritsch, en Amazonas, Huanuco, Loreto, Madre de Dios, San Martín y Ucayali hasta los $1000 \mathrm{msnm}$; y $C$. crassifolia (Focke) C.V. Morton, en Huanuco, Loreto y San Martín hasta los 2000 msnm (Brako \& Zarucchi, 1993; Ulloa et al., 2004). Estas plantas se caracterizan por tener nectarios extraflorares, por lo que es común encontrar hormigas pecoreando sobre éstos; y también es posible encontrar individuos epífitos creciendo sobre los nidos colgantes de algunas especies de estos insectos, recibiendo el nombre de “jardines de hormigas” (Davison, 1989; Montero et al., 2003; Soley et al., 2004).

Publicaciones sobre Gesneriaceae en el Perú podemos empezar citando a Skog (1982) sobre las especies de esta familia para Perú y Ecuador; además tenemos a Vásquez (1997) que en las reservas naturales de Iquitos-Loreto registró 73 especies de las cuales 3 fueron del género Codonanthe (C. crassifolia, C. uleana y C. sp.). También el trabajo de Hernani (2006) en el Parque Nacional Yanachaga Chemillén- 
Pasco donde se reportaron 63 especies, 36 de las cuales son nuevos registros para Pasco. Finalmente podemos citar un trabajo reciente de Clark et al. (2005) en el que se describe una nueva especie para Perú: Alloplectus carpishensis J.L. Clark \& I. Salinas. De estos últimos trabajos se puede deducir que las gesneriáceas no han sido suficientemente colectadas. En la zona del río Los Amigos, se colectaron 17 morfoespecies de la misma familia de las cuales 3 son de Codonanthe, pero ninguna aparece determinada (BRIT, 2005).

Según Brack (1999) estas especies son utilizadas como plantas medicinales por indígenas y colonos de la amazonía peruana. Por ejemplo C. crassifolia "shanen bana" es utilizada para tratar los dolores de cabeza con la decocción de las hojas. C. uleana "kanatépa” es usada por los Ticuna para tratar heridas e infecciones recalcitrantes, en base a sus hojas machacadas; sin embargo los colonos la llaman "picsho" y la usan además, para edemas.

Ecológicamente se sabe que estas plantas coexisten con hormigas. Davinson (1989), en Cocha Cashu (Parque Nacional del Manu-Madre de Dios) quien reportó a $C$. uleana creciendo sobre jardines de hormigas de las especies Azteca cf. traili, Camponotus femoratus, Crematogaster cf. limata parabiotica, Solenopsis sp., Pheidole sp1., Pheidole sp. 2 y Odontomachus haematodus; acompañadas además por otras epífitas como Anthurium gracile, Philodendron megalophyllum, Neoregelia sp., Streptocalyx longifolius, Epidendrum phyllanthus, Ficus paraensis, Peperomia macrostachya y Markea ulei. En el río Los Amigos también existen trabajos similares a este realizados por Montero et al. (2003) y otros por Soley et al. (2004), ambos orientados a intentar explicar la riqueza de epífitas en estos jardines, en los cuales se reporta a Camponotus femoratus y Crematogaster cf. limata parabiotica en asociación con estas epífitas. Además, se menciona la interacción parabiótica entre las hormigas Camponotus femoratus y Crematogaster cf. limata parabiotica, quienes coexisten en el mismo nido, esto para ambas localidades (Cocha Cashu y Los Amigos) y siempre en interacción con C. uleana.

En nuestro país son escasos los estudios sobre plantas epífitas en general, pese a que se estima que un $11 \%$ de nuestras plantas vasculares son epífitas (Nieder et al., 2001) y que en el resto del mundo el $10 \%$ de todas estas (como unas 25000 especies) también son epífitas (Nieder et al., 1997, 2001; Catchpole, 2004). Por lo tanto, debido a los vacíos de información en este aspecto el presente trabajo tiene el objetivo de contribuir al conocimiento ecológico y taxonómico de las plantas epífitas en el Perú.

\section{Materiales y métodos}

Área de Estudio

Las observaciones se realizaron en la cuenca del río Los Amigos, el cual es el límite político entre las provincias de Manu y Tambopata en el departamento de Madre de Dios (Figura 1). El centro de operaciones fue la estación biológica CICRA (ubicada 12³4'06.9 " LS; 7005'56.5" LW) al encontrarse esta en un sitio estratégico de la cuenca y por sus comodidades de alojamiento. Según el Mapa Ecológico del Perú (INRENA, 1995), la zona de vida corresponde a un bosque húmedo tropical de transición a subtropical (bh-T/S). Basándonos en el sistema de clasificación propuesto por Encarnación (1993) para los tipos de bosques y otras formaciones vegetales de la Llanura Amazónica peruana, podemos diferenciar con facilidad tres formaciones boscosas muy bien delimitadas para esta zona: El Aguajal: con dominancia de palmeras de aguaje (Mauritia flexuosa), se encuentran en depresiones de otros bosques de terrenos bajos, generalmente lejos de las masas de agua blanca y casi siempre inundadas por aguas negras o de mezcla debido al drenaje casi nulo de sus suelos; además hay escasa diversidad de árboles, de los cuales ninguno es emergente. Bosque de Llanura Baja: conocido como Bajío, está conformado por bosques inundables de mayor extensión en la llanura amazónica; se caracterizan por inundarse con facilidad ante cualquier lluvia fuerte; tienen niveles freáticos bastante altos, suelos con niveles variables de drenaje, y altos niveles de descomposición de materia orgánica; además hay árboles emergentes y una alta diversidad de epífitos. Bosque de Terraza Alta: se establecen sobre terrenos planos alejados de los ríos; suelos arcillosos o arenosos con drenaje mediano a bueno por lo que nunca se inundan a causa de las lluvias, además hay árboles emergentes, pero una pobre diversidad de epífitas.

\section{Procedimientos}

El trabajo de campo estuvo basado en colecciones botánicas a lo largo del sistema de trochas de la estación biológica CICRA y fuera de éste; haciendo siempre anotaciones sobre el tipo de hábitat circundante y el modo de crecimiento de los especimenes. Como las plantas del género Codonanthe normalmente están asociadas con hormigas se dio especial énfasis a localizarlas en estos nidos que son también llamados "jardines de hormigas”. El proceso de herborización del material botánico colectado se realizó por los métodos convencionales. Además se colectaron especimenes vivos para adaptarlos al cultivo y de esa forma poder contar con material fresco para su revisión posterior. Estos se encuentran albergados en el vivero del Programa de Horticultura "EL HUERTO" de la Universidad Nacional Agraria La Molina. El sustrato utilizado para ello estuvo conformado por musgo fino y compost; protegiendo además a las plantas del frío con un túnel de plástico que se les instalaba por las noches. 


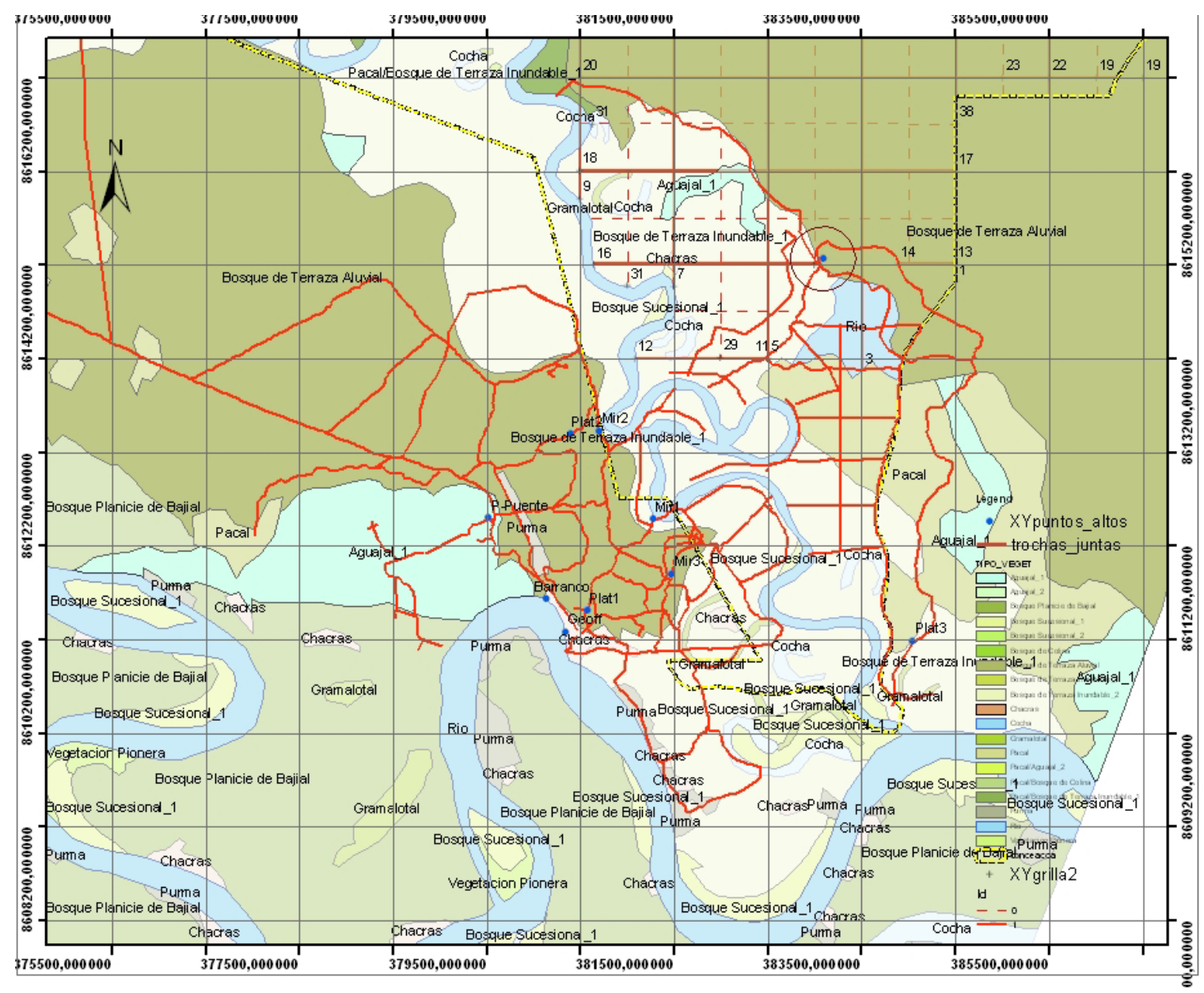

Figura 1: Mapa de ubicación de la zona de estudio, mostrando las principales formaciones vegetales y el actual sistema de trochas de la estación experimental CICRA (cortesía de la WWF).

Para las determinaciones taxonómicas, se recurrió al uso de claves como las publicadas por Skog \& Kvist (1997) y Vásquez (1997). Así mismo, se compararon las muestras con otras colecciones depositadas en el herbario MOL de la Universidad Nacional Agraria La Molina y en el herbario USM de la Universidad Nacional Mayor de San Marcos. Además se visitó los sites de "W3 TROPICOS" de Missouri Botanical Garden (2006) y “ATRIUM” del Botanical Research Institute of Texas (2005) donde se hizo uso de la base de datos y una galería fotográfica disponibles.

\section{Resultados y Discusión}

El género Codonanthe se caracteriza por estar formado principalmente por hierbas epífitas, aunque también existen especies arbustivas y otras de hábito lianescente; para nuestro país todas epífitas, siendo común encontrarlas creciendo en nidos de hormigas. Los tallos son erguidos, péndulos o reptantes, y a veces con raíces adventicias. Las hojas se caracterizan por ser muy suculentas y crasas (vegetativamente es muy fácil confundirla con una crassulácea), generalmente con nectarios extraflorales, razón por la cual es frecuente encontrar a estas plantas asociadas con hormigas. Inflorescencias axilares, solitarias o paucifloras, en fascículos. Flores bracteadas; cáliz 5lobulado, actinomorfa o zigomorfa; corola blanca o coloreada y generalmente con líneas o puntos rojizos, infundibuliforme o subcampanulada, a veces espolonada en la base y comprimida, 5-lobulada; estambres 4; disco compuesto de una glándula; ovario súpero, estigma 2-lobulado. Fruto cápsula abayada, tardíamente dehiscente, 2- valvada, globosa, ovoide o comprimida, roja, rosada, anaranjada o amarilloverdosa. Para la zona del río Los Amigos encontramos C. crassifolia y C. uleana:

Clave de determinación de las especies

1. Hojas enteras; estambres con filamentos ca. $8 \mathrm{~mm}$ de largo, anteras sin aristas ............. C. crassifolia 1'. Hojas usualmente dentadas o crenadas hacia el ápice o raramente enteras; estambre con filamentos ca. $20 \mathrm{~mm}$ de largo, anteras con dos aristas ..... C. uleana 
Descripción de las Especies

Codonanthe crassifolia (H. Focke) C.V. Morton

Hierbas o enredaderas epífitas, glabras. Hojas elípticas, ápice agudo, base obtusa o redondeada, margen entero. Flores solitarias, pedúnculos 5-8 mm de largo; cáliz 4-6 mm de largo, lóbulos lineares; corola blanca, infundibuliforme, glabra por fuera, tubo 20-23 mm de largo, espolonada, 5-lóbulada, lóbulos obtusos. Fruto abayado de color rojo o grosella ca. 1 $\mathrm{cm}$ de diámetro, que se abre en 2 mitades cuando madura por lo que algunos lo consideran una cápsula. Material revisado: M. S. Vega 434 (MOL), 2004; M. S. Vega 453 (MOL), 2004; M. A. Chocce 428 (BRIT), 2004.

Distribución y ecología: Esta especie tiene una amplia distribución en todo el neotrópico (Figura 2) aunque en Los Amigos es poco frecuente; incluso uno de los especimenes se le colectó accidentalmente en una rama caída de un árbol de Cedrelinga sp. (Fabaceae) de aproximadamente $35 \mathrm{~m}$ de altura, por lo que se presume que podrían existir poblaciones de esta especie colonizando el dosel superior del bosque, es decir, por lo menos, las zonas 3 y 4 tipificadas por Johansson (1974) las que comprenden la base de la copa y la copa media del árbol. Los especimenes colectados se encontraron en la terraza y en el aguajal, en zonas que reciben abundante luz solar, lo que sugiere que esta especie tiende a buscar bastante luz para sus requerimientos fisiológicos (Daubenmire, 1990; Vickery, 1991; Córdoba \& Legas, 2000).

Se adapta muy bien al cultivo y al clima de Lima, de modo que después de un tiempo de adecuación al cultivo ya no es necesario seguir protegiendo las plantas por las noches con el túnel. Como su aspecto vegetativo, sus flores y frutos son muy atractivos podría considerarse como un potencial recurso ornamental (Figura 3).

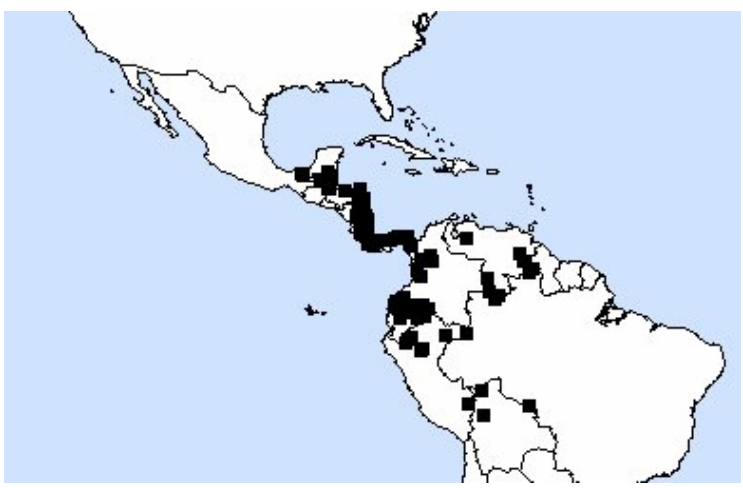

Figura 2: Mapa de puntos de colecta para especimenes de Codonanthe crassifolia (H. Focke) C.V. Morton (fuente W3 TROPICOS).

No se reportan colecciones en el área de estudio para esta especie sobre jardines de hormigas. Además no se reportan otras colecciones de esta especie para
Madre de Dios, salvo el voucher M. A. Chocce 428 en el BRIT, pero que no fue determinado como $C$. crassifolia, por lo que este sería el primer reporte para la flora de Madre de Dios, ampliando su distribución.

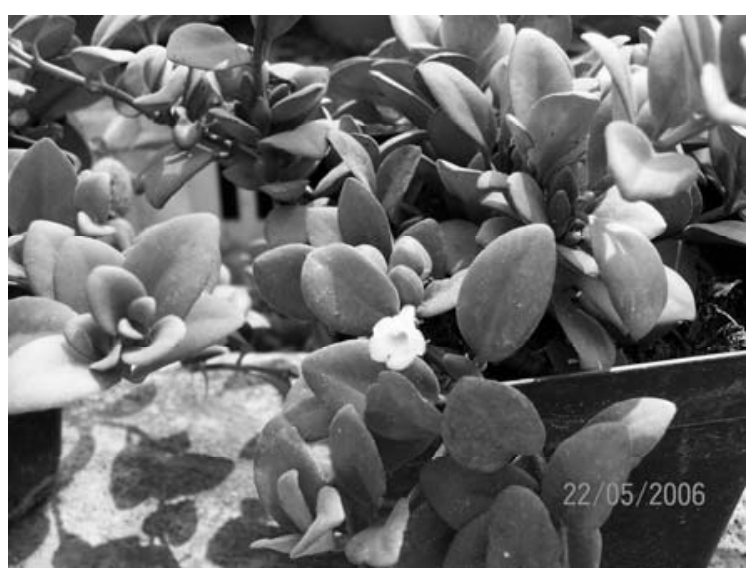

Figura 3: Codonanthe crassifolia (H. Focke) C.V. Morton en cultivo (foto Michael S. Vega).

\section{Codonanthe uleana Fritsch}

Hierbas epífitas, glabras. Hojas elíptico - obovadas u oblanceoladas de color verde rojizo o verde claro según la exposición a la luz, ápice acuminado o agudo, base cuneada o decurrente, margen dentado o crenado hacia el ápice (entero); peciolos 3-15 mm de largo. Flores solitarias, pedúnculos 5-7 mm de largo; cáliz 3$4 \mathrm{~mm}$ de largo, lóbulos lineares; corola blanca o con líneas rosadas, infundibuliforme, glabra por fuera, tubo 22-35 mm de largo, espolón ca. $3 \mathrm{~mm}$ de largo, redondeado. Fruto abayado de color rosado o grosella ca. $1 \mathrm{~cm}$ de diámetro, que se abre en 2 mitades cuando madura por lo que algunos lo consideran una cápsula. Material revisado: M. S. Vega 373 (MOL), 2004; M. S. Vega 541 (MOL), 2004; M. A. Chocce 629 (BRIT), 2004; M. A. Chocce 717 (BRIT), 2004.

Distribución y ecología: Esta especie también tiene una amplia distribución en todo el neotrópico (Figura 4), y además es muy abundante en Los Amigos y aparentemente específica para los jardines de hormigas, ya que sólo se encontró un individuo fuera de estos en el aguajal. En los jardines de hormigas se le encontró asociada con las especies Azteca sp., Camponotus femoratus y Crematogaster cf. limata parabiotica, que además fueron las únicas especies de hormigas que forman estos jardines con epífitas.

En los jardines parabióticos de Camponotus femoratus y Crematogaster cf. limata parabiotica se le observó creciendo en compañía de otras epífitas de las especies Anthurium gracile, Philodendron megalophyllum, Epidendrum phyllanthus, Ficus paraensis, Ficus sp., Peperomia macrostachya, Markea cf formicarum y una bromeliácea indeterminada (Figura 7); muchas de las cuales son especies también reportadas en otras investigaciones 
(Davinson, 1989; Montero et al., 2003; Soley et al., 2004). Estos jardines parabióticos son particularmente abundantes en la terraza y en el bajío, aunque también se logró encontrar 2 de estos en el aguajal; todos estos siempre con C. uleana; comúnmente cerca de claros en los estrados bajos del bosque, pues es raro ver este tipo de jardines por encima de los $15 \mathrm{~m}$.

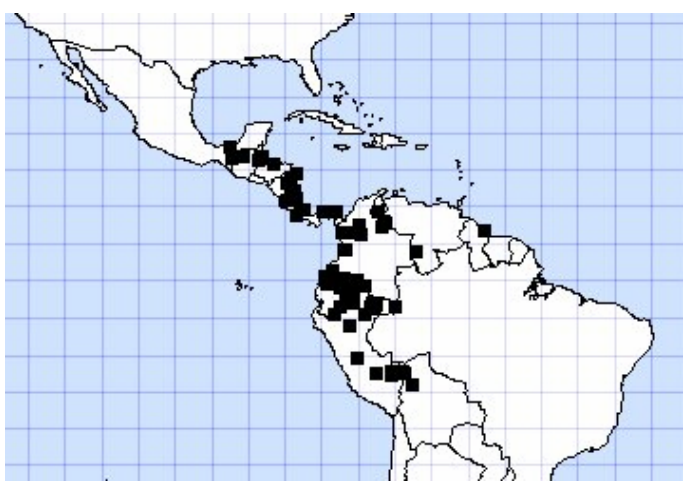

Figura 4: Mapa de puntos de colecta para especimenes de Codonanthe uleana Fritsch (fuente W3 TROPICOS).

En los jardines de Azteca sp. encontramos a esta especie creciendo como epífita exclusiva, ya que no se encontraron otras epífitas acompañándola. Estos jardines son muy abundantes en el aguajal, sobre todo encima de los arbustos de Tococa guianensis y Erithroxylum sp.; logrando encontrar sólo uno de estos en la terraza bajo la copa de un arbolillo de Hirtella racemosa; pero siempre por debajo de los $5 \mathrm{~m}$ de altura. Justamente en esta clase de jardines se observó una modificación morfológica producto de la cantidad de luminosidad que la planta recibe en su hábitat; pues al parecer cuando $C$. uleana crece en lugares con abundante cantidad de luz (como lo son los aguajales y los claros para el caso de los jardines parabióticos de Camponotus femoratus y Crematogaster cf. limata parabiotica) sus hojas son de un color verde oscuro con tonos rojizos y muy suculentas (Figura 5), mientras que cuando la planta crece en lugares con mayor sombra (como en el caso del jardín de Azteca sp. que estaba en la terraza) sus hojas son más alargadas, menos suculentas y de un color verde claro muy intenso (Figura 8). Estos dos morfotipos, sí se observan por separado en el campo podrían ser interpretados fácilmente como especies diferentes; no obstante, se tratan únicamente de adaptaciones que los individuos de esta especie hacen en función a las variaciones de luminosidad del hábitat (Daubenmire, 1990; Vickery, 1991; Córdoba \& Legas, 2000).

Para su cultivo es recomendable mantener a la planta siempre en lugar abrigado para garantizar su crecimiento (Figura 6). Justamente gracias a esto fue posible notar que los dos morfotipos descritos en el párrafo anterior eran de la misma especie.

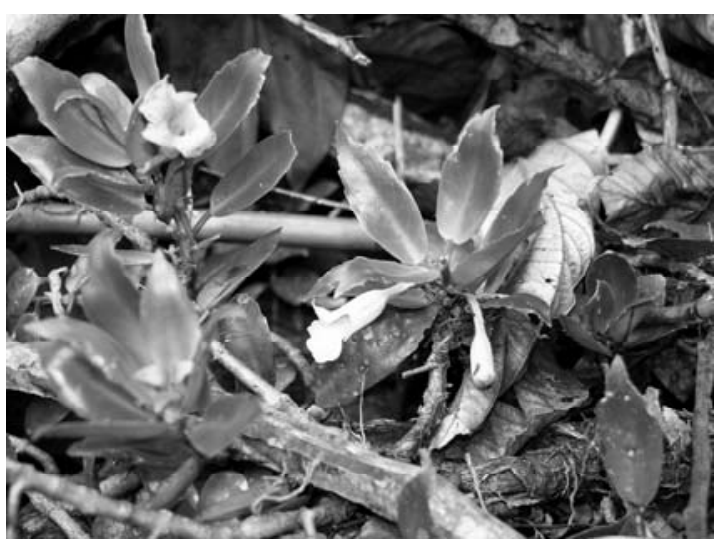

Figura 5: Codonanthe uleana Fritsch en hábitat (foto Michael S. Vega).

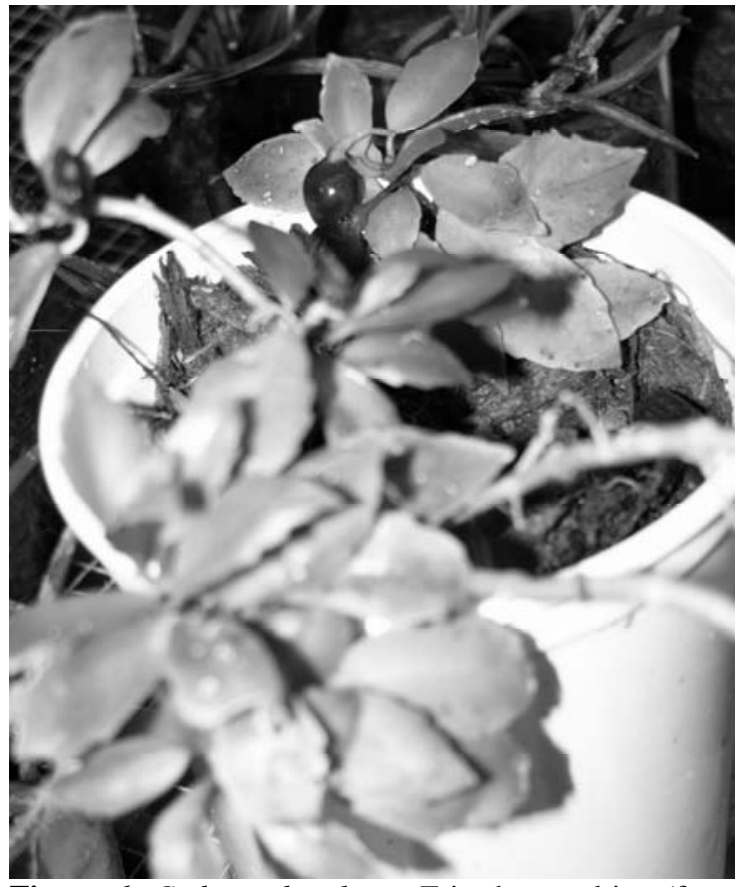

Figura 6: Codonanthe uleana Fritsch en cultivo (foto Michael S. Vega).

\section{Conclusiones}

1. Se reportan 2 especies de Codonanthe para la cuenca del río Los Amigos: C. crassifolia y $C$. uleana. De estas, C. crassifolia constituye un nuevo reporte para la flora de Madre de Dios.

2. C. crassifolia es una especie de raro avistamiento en Los Amigos; aunque aun es necesario hacer más investigaciones sobre su estado poblacional.

3. C. uleana fue la única especie encontrada en asociación con hormigas, la cual estuvo creciendo sobre los jardines de Azteca sp. principalmente en el aguajal, y en los parabióticos de Camponotus femoratus y Crematogaster cf. limata parabiotica 
principalmente en la terraza y en el bajio (Figura 7 y 8).

4. Como flora acompañante de C. uleana se encontraron a Anthurium gracile, Philodendron megalophyllum, Epidendrum phyllanthus, Ficus paraensis, Ficus sp., Peperomia macrostachya, Markea cf formicarum y una bromeliácea indeterminada; siempre en los jardines parabióticos de Camponotus femoratus y Crematogaster cf. limata parabiotica. En los nidos de Azteca sp. crecía como epífita exclusiva.

5. En C. uleana, se observaron dos morfotipos: a). Bajo luz intensa o directa, y b). Bajo luz difusa o sombra; que pueden ser confundidos como dos especies distintas.

6. Estos morfotipos en C. uleana son sólo adaptaciones específicas a la cantidad de luz disponible, por lo que no los consideramos como variaciones de importancia taxonómica.

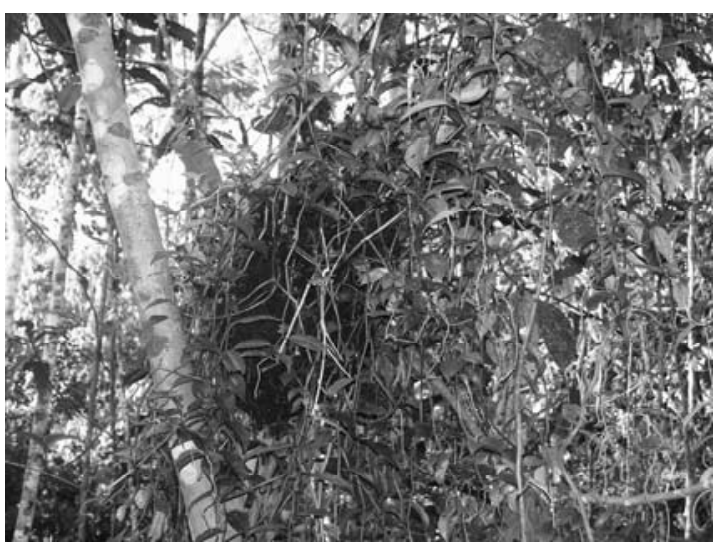

Figura 7: Jardín de Hormigas de las especies Camponotus femoratus y Crematogaster cf. limanata parabiotica en un bosque de terraza (foto Michael S. Vega).

\section{Agradecimientos}

Gracias especiales para el Sr. Remigio Yumpata y a la Dra. Graciela Vilcapoma. A los Ingenieros Saray Siura y Roberto Ugás de la UNALM; a las profesoras Mag.Sc. Joaquina Alban y Mag.Sc. María Isabel La Torre del herbario USM. También al Dr. Nigel Pitman, Dra. Reneta Leite, Ing. Jorge Herrera y al resto del personal que trabaja en el CICRA. Al Dr. Jonh Janovec, Ing. Fernando Cornejo, Blgo. Miguel Chocce y Piher Maceda del BRIT. A Pedro Centeno, Blga. Lizbeth Hernani (herbario HUSA), Dra. Elsa Youngs (North Carolina State University) y Blgo. Raúl Tupayachi (WWF). A Johanna Cortez y Alexander Blaz (UNALM). Finalmente a ACCA por el financiamiento otorgado.

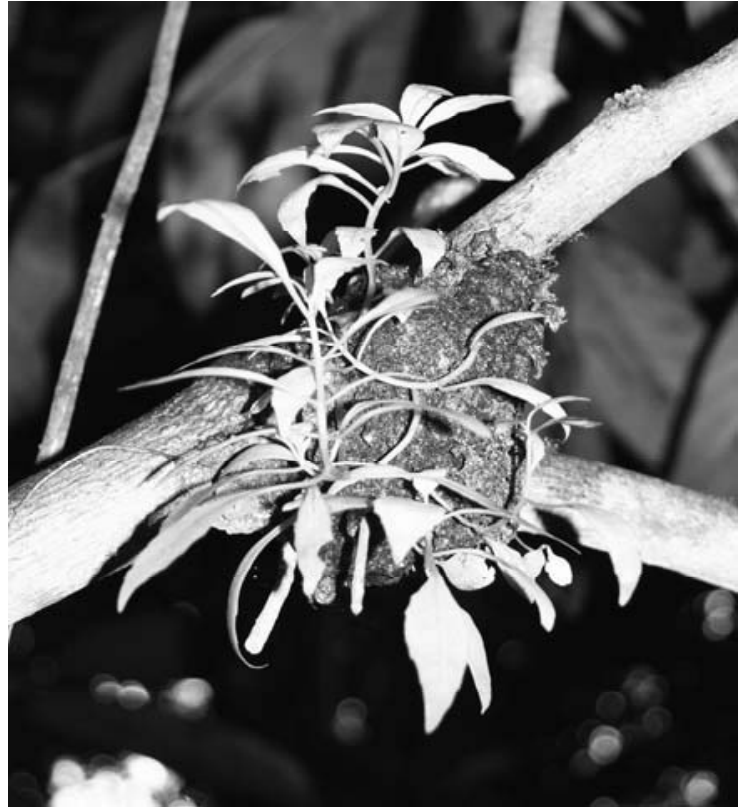

Figura 8: Codonanthe uleana Fritsch en jardín de Azteca sp. en el bosque de terraza (foto Michael S. Vega).

\section{Literatura citada}

Brako L. \& Zarucchi J.L. 1993. Catalogue of the Flowenig Plants and Gymnosperms of Peru. Missouri Botanical Garden. St. Louis, USA.

Brack A. 1999. Diccionario Enciclopédico de las Plantas Útiles del Perú. CBC. Cuzco, Perú.

Botanical Research Institute of Texas (BRIT). 2005. ATRIUM: The Biodiversity Information System. En: http://atrium.andesamazon.org/

Catchpole D. 2004. The Ecology of Vascular Epiphytes on Ficus L. Host (Moraceae) in a Peruvian Cloud Forest. Tesis de Magíster - Universidad de Tasmania, Australia.

Clark J.L., Salinas I. \& Skog L.E. 2005. Novae Gesneriaceae Neotropicarum XIV: four new species of Alloplectus (Gesneriaceae) from South America. Novon. 15: 70-79.

Córdoba C. \& Legas M. 2000. Fisiología Vegetal Ambiental. Editorial Síntesis S. A. Madrid, España.

Daubenmire R.F. 1990. Ecología Vegetal: Tratado de Autoecología de Plantas. Editorial Limusa. México.

Davidson D.W. 1989. Ecological Studies of Neotropical Ant Gardens. Ecology. 69 (4): 1138- 152.

Encarnación F. 1993. El Bosque y las Formaciones Vegetales en la Llanura Amazónica del Perú. ALMA MATER - UNMSM.: 95-114.

Hernani L. 2006. Diversidad y Distribución de Gesneriaceae Rich. \& Juss. en tres sectores del Parque Nacional Yanachaga - Chemillén , Oxapampa, Pasco Perú, Marzo - Abril, 2006. En: Anales del XI Congreso Nacional de Botánica. Universidad Nacional del Altiplano. Puno, Perú.

Johansson D.R. 1974. Ecology of Vascular Epiphytes in West African Rainforest. Acta Phytogeographyca. Suecia. 
Kvist L.P., Skog L.E., Clark J.L. \& Dunn R.W. 2004. The family Gesneriaceae as example for the Biological extinction in Western Ecuador. Lyonia. 6(2): 127- 51.

Missouri Botanical Garden (MO). 2006. W3 TROPICOS. En: http://mobot.mobot.org/W3T/Search/vast.html

Montero A., Estrada N. \& Paiz Y. 2003. ¿Qué Factores afectan la Riqueza de Epífitas en Jardines de Hormigas? En: Ecología de Ecosistemas Amazónicos, OTS 2003. Puerto Maldonado, Perú.

Nieder J., Prosperí J. \& Michaloud G. 2001. Epiphytes and their contribution to canopy diversity. Plant Ecology. 153(1-2): $51-63$.

Nieder J., Ibisch P.L. \& Barthlott W. 1997. Biodiversidad de Epífitas - Una cuestión de escala. Revista del Jardín Botánico Nacional. XVII - XVIII. : 59-62.

INRENA. 1995. Mapa Ecológico del Perú: Guía Explicativa. Lima, Perú.

Skog L.E. 1982. New Gesneriaceae from Peru and Equador. Selbyana. 7: 94-99.

Skog L.E. \& Kvist L.P. 1997. The Gesneriaceae of Ecuador. En: Valencia, R. \& Balslev, H. (eds.).
Estudios sobre diversidad y ecologia de plantas; Memorias del II Congreso Ecuatoriano de Botánica. Pontificia Universidad Catolica del Ecuador, Quito.

Soley F., Artavia L.G. \& Sánchez C. 2004. Cambios en la Composición y Riqueza de Epífitas en Jardines de Hormigas de distinta Edad y Tamaño. En: Ecología de Ecosistemas Amazónicos, OTS 2004. Puerto Maldonado, Perú.

Ulloa C., Zarucchi J. \& León B. 2004. Diez Años de Adiciones a la Flora Peruana, 1993 - 2003. En: Arnaldoa, Edición Especial Noviembre 2004. Trujillo, Perú.

Vásquez R. 1997. Flórula de las Reservas Biológicas de Iquitos, Perú. Missouri Botanical Garden. St. Louis, USA.

Vickery M.L. 1991. Ecología de Plantas Tropicales. Editorial Limusa. México.

Wiehler H. 1983. A synopsis of the neotropical Gesneriaceae. Selbyana. 6: 1-219.

1'Jardín Botánico “Octavio Velarde Núñez”, Universidad Nacional Agraria La Molina. 20000157@lamolina.edu.pe / aceroni@lamolina.edu.pe. Apartado postal: 12-056., Lima 12, Perú.

${ }^{2}$ Docente de la Facultad de Ciencias Forestales, Universidad Nacional Agraria La Molina. Apartado postal 12056 Lima 12. Correo electrónico: reynel@lamolina.edu.pe 